QUARTERLY OF APPLIED MATHEMATICS

VOLUME LXIX, NUMBER 2

JUNE 2011, PAGES 389-401

S 0033-569X(2011)01214-X

Article electronically published on March 10, 2011

\title{
ASYMPTOTIC SOLUTION OF A NONLINEAR ADVECTION-DIFFUSION EQUATION
}

\author{
BY \\ R. DE LOUBENS (Schlumberger-Doll Research, 1 Hampshire Street, Cambridge, Massachusetts \\ 02139) \\ AND \\ T. S. RAMAKRISHNAN (Schlumberger-Doll Research, 1 Hampshire Street, Cambridge, \\ Massachusetts 02139)
}

Abstract. We carry out an asymptotic analysis as $t \rightarrow \infty$ for the nonlinear advectiondiffusion equation, $\partial_{t} u=2 \alpha u \partial_{x} u+\partial_{x}\left(u \partial_{x} u\right)$, where $\alpha$ is a constant. This equation describes the movement of a buoyancy-driven plume in an inclined porous medium, with $\alpha$ having a specific physical significance related to the bed inclination. For compactly supported initial data, the solution is characterized by two moving boundaries propagating with finite speed and spanning a distance of $\mathcal{O}(\sqrt{t})$. We construct an exact outer solution to the PDE that satisfies the right boundary condition. The vanishing condition at the left boundary is enforced by introducing a moving boundary layer, for which we obtain a closed-form expression. The leading-order composite solution is uniformly correct to $\mathcal{O}(1 / \sqrt{t})$. A higher-order correction to the inner and the composite solutions is also derived analytically. As a result, we obtain late-time asymptotic expansions for the two moving boundaries, correct to $\mathcal{O}(1)$, as well as a composite solution correct to $\mathcal{O}(1 / t)$. The findings of this paper are illustrated and verified by numerical computations.

1. Introduction. We consider the initial value problem (IVP) defined by

$$
\begin{gathered}
\frac{\partial u}{\partial t}=2 \alpha u \frac{\partial u}{\partial x}+\frac{\partial}{\partial x}\left(u \frac{\partial u}{\partial x}\right), \\
u(x, 0)=u_{I}(x),
\end{gathered}
$$

where $\alpha$ is a constant. The initial condition $u_{I}$ is nonnegative, with unit mass, and has a compact support.

The degenerate parabolic PDE (1.1) has multiple applications in porous media flow, e.g. in water infiltration theory [3, 11]. More recently, it has been shown to govern the interface shape evolution of a gravity tongue propagating up an inclined porous layer [9] with respect to a moving frame. Here $u$ is the thickness of the gravity tongue, and $\alpha>0$ is a parameter proportional to the up-dip slope and the mobility ratio of the lighter to

Received September 23, 2009.

2000 Mathematics Subject Classification. Primary 35-XX. 
the heavier fluid. By reversing $x$, the analysis applies to the opposite case of a plume migrating down-dip.

Theoretical proofs of existence, uniqueness and regularity of the solution to the Cauchy problem (1.1)-(1.2) are provided in [2]. It was also shown by Gilding [3] that the solution $u(x, t)$ conserves mass, i.e.,

$$
\int_{\mathbb{R}} u(x, t) d x=1, \quad t \geq 0 .
$$

Except in the special case $\alpha=0$, where a self-similar analytical solution can be constructed for an instantaneous fluid release [1], (1.1) generally does not admit an exact solution. In [5, 7, 8, late-time solutions have been explored for a more general form of (1.1), given by

$$
\frac{\partial u}{\partial t}=\frac{\partial\left(u^{q}\right)}{\partial x}+\frac{\partial^{2}\left(u^{m}\right)}{\partial x^{2}},
$$

where $q$ and $m$ are fixed exponents. For $1<m<2 q-1$, Gilding [3] showed that there exists a unique weak solution of (1.4), and that it is characterized by a finite speed of propagation of a left moving boundary and a right moving boundary, defined as

$$
\begin{aligned}
& x_{\ell}(t)=\inf \{x \in \mathbb{R}: u(x, t)>0\}, \\
& x_{r}(t)=\sup \{x \in \mathbb{R}: u(x, t)>0\} .
\end{aligned}
$$

Gilding [4] proved the existence of frontal solutions for an even more general form than (1.4). When his results are applied to the special case (1.4), we obtain the existence of (1.5) and (1.6) for $m>1$ and $q \geq 1$. The evolution of the support of solutions to multi-dimensional convection-diffusion problems has also been studied, an example of which is the work of Sapronov and Shishkov [10].

Based on asymptotic expansions, Grundy [5] derived closed-form expressions for the leading-order outer solution of (1.4). For $q-1<m \leq q$ and $m>1$, he proposed a boundary layer on both edges of the solution. For late-time convection dominated flow $(m>1$ and $q \in(1, m+1)$ or $m \geq 1$ and $q \in(0,1))$, Laurençot [7, 8] showed that with an appropriate $L^{p}$-norm, $p<\infty$, the true solution asymptotically converges to the hyperbolic limit.

In this paper, we derive both outer and inner expansions to the problem stated in (1.1) and (1.2). A composite leading-order solution valid for $x_{\ell}(t) \leq x \leq x_{r}(t)$, correct to $\mathcal{O}(1 / \sqrt{t})$, is also obtained. The leading-order inner and outer solutions are consistent with those of Grundy [5], but our results do not indicate a right boundary layer. One has to account for the movement of the boundary through an appropriate expansion, without which a matched solution cannot be obtained. We show that with $q=m=2$, there exists an exact solution to (1.1). This solution satisfies the right boundary condition, which also confirms that no boundary layer is required at the leading edge. Higher-order boundary layer solutions at the trailing edge are given along with the results for the composite solution, valid to $\mathcal{O}(1 / t)$. At this order, an unknown constant appears in the solution. Numerical computations that support these conclusions are presented. 
2. Zeroth-order solution. We first seek a late-time similarity solution of the form

$$
u^{\mathbf{o}}(x, t)=t^{a} f(\eta), \quad \eta=x t^{b},
$$

where $a$ and $b$ are unknown exponents and $f$ is a nonnegative $\mathcal{O}(1)$ function. From Gilding's theorem [2], we have to satisfy the condition that $u=0$ at $x=x_{\ell}(t)$ and $x=x_{r}(t)$, where $x_{\ell}(t) \rightarrow-\infty$ and $x_{r}(t) \rightarrow+\infty$ as $t \rightarrow \infty$. Substitution of (2.1) for $u$ into (1.1) leads to the second-order ODE

$$
a f+b \eta f^{\prime}=\frac{1}{2} t^{a+2 b+1}\left(f^{2}\right)^{\prime \prime}+\alpha t^{a+b+1}\left(f^{2}\right)^{\prime} .
$$

From the mass conservation condition, $\int_{-\infty}^{\infty} u(x, t) d x=1$. Since (2.1) is a late-time similarity solution, the integral condition is to be applied asymptotically. Hence, with $\eta_{\ell}$ and $\eta_{r}$ as the left and right $\eta$ corresponding to $x_{\ell}$ and $x_{r}$, respectively, we impose that

$$
1=\int_{-\infty}^{\infty} t^{a} f\left(x t^{b}\right) d x+\cdots=t^{a-b} \int_{\eta_{\ell}(t)}^{\eta_{r}(t)} f(\eta) d \eta+\cdots .
$$

With $\eta=\mathcal{O}(1)$ and $f(\eta)=\mathcal{O}(1)$, we see that

$$
a=b,
$$

a result also obtained by Grundy [5]. Thus (2.2) becomes

$$
a f+a \eta f^{\prime}=\frac{1}{2} t^{3 a+1}\left(f^{2}\right)^{\prime \prime}+\alpha t^{2 a+1}\left(f^{2}\right)^{\prime} .
$$

The above left-hand side is $\mathcal{O}(1)$. Regardless of which term from the right-hand side is dominant, $a<0$. For a distinguished limit it is then obvious that

$$
2 a+1=0 \Rightarrow a=-\frac{1}{2} .
$$

2.1. Outer solution. We now seek an expansion of the form

$$
u^{\mathbf{o}}(x, t)=\frac{1}{\sqrt{t}}\left(f_{0}(\eta)+o(1)\right), \quad \eta=\frac{x}{\sqrt{t}},
$$

where $f_{0}$ is the leading-order representation of $f$. We also denote by $\eta_{\ell 0}$ and $\eta_{r_{0}}$ the unknown zeroth-order left and right boundary positions. Keeping only the $\mathcal{O}(1)$ terms in the differential equation (2.5), i.e., dropping the second derivative term on the right-hand side, we obtain

$$
2 \alpha\left(f_{0}^{2}\right)^{\prime}+\eta f_{0}^{\prime}+f_{0}=0 .
$$

Integration of this ODE yields

$$
2 \alpha f_{0}^{2}+\eta f_{0}=C_{1},
$$

where $C_{1}$ is a constant. To satisfy the boundary condition at the left or the right edge, we need $C_{1}$ to be zero. Therefore,

$$
f_{0}(\eta)=-\frac{\eta}{2 \alpha} \vee 0
$$

Since $f_{0} \geq 0$, the first solution is valid for $\eta \leq 0$. It follows that

$$
\eta_{r_{0}}=0 \text {. }
$$


Using the mass conservation to zeroth-order,

$$
\int_{\eta_{\ell 0}}^{0} f_{0}(\eta) d \eta=1
$$

we obtain $\eta_{\ell 0}=-2 \sqrt{\alpha}$, and conclude that

$$
f_{0}(\eta)= \begin{cases}-\frac{\eta}{2 \alpha}, & -2 \sqrt{\alpha} \leq \eta \leq 0, \\ 0, & \eta \geq 0, \quad \eta \leq-2 \sqrt{\alpha} .\end{cases}
$$

This solution conforms to the result of [5] for $\alpha=1$. We note that $u_{0}^{\mathbf{o}}(x, t)=f_{0}(x / \sqrt{t}) / \sqrt{t}$ is the exact solution to the hyperbolic limit of (1.1) for the initial condition $u_{I}(x)=$ $\delta(x)$, where $\delta(x)$ is the Dirac distribution centered at zero. We refer to Laurençot and Simondon [7 for a proof of asymptotic convergence to the hyperbolic limit solution for a scaled $L^{p}$-norm with $p \in[1, \infty)$.

A discontinuity at $\eta=-2 \sqrt{\alpha}$ implies a boundary layer at the left where the secondorder derivative in (2.5) becomes important.

2.2. Inner solution. A boundary layer is constructed by seeking an inner expansion about $\eta_{\ell 0}=-2 \sqrt{\alpha}$. We introduce the "stretched" coordinate

$$
\xi_{0}=\left(\eta-\eta_{\ell 0}\right) t^{d},
$$

where $d$ is a positive exponent yet to be determined. With $\xi_{0}=\mathcal{O}(1)$, the scale of $u(x, t)$ being $\frac{1}{\sqrt{t}}$ as in the outer solution, we write

$$
u^{\mathbf{i}}(x, t)=\frac{1}{\sqrt{t}} g_{0}\left(\xi_{0}\right)+\cdots,
$$

where $g_{0}$ is an $\mathcal{O}(1)$ function in the boundary layer. It is important to reemphasize that at this stage, $\eta_{\ell}$ is known only to leading order $\eta_{\ell 0}=-2 \sqrt{\alpha}$.

After substitution into (1.1), we obtain

$$
-\left((1-2 d) \xi_{0}+\eta_{\ell 0} t^{d}\right) g_{0}^{\prime}-g_{0}=2 \alpha t^{d}\left(g_{0}^{2}\right)^{\prime}+t^{2 d-\frac{1}{2}}\left(g_{0}^{2}\right)^{\prime \prime}+\cdots .
$$

In the boundary layer, we satisfy the second boundary condition of $u=0$. Unlike the outer solution formulation, the second derivative must be accounted for. Therefore, the distinguished limit is obtained by necessitating the presence of second-order derivatives on the right-hand side of (2.16), or

$$
d=2 d-\frac{1}{2} \Rightarrow d=\frac{1}{2} .
$$

The leading-order inner solution then satisfies

$$
\left(g_{0}^{2}\right)^{\prime \prime}+2 \alpha\left(g_{0}^{2}\right)^{\prime}-2 \sqrt{\alpha} g_{0}^{\prime}=0,
$$

implying that

$$
\left(g_{0}^{2}\right)^{\prime}+2 \alpha g_{0}^{2}-2 \sqrt{\alpha} g_{0}=C_{2},
$$

where $C_{2}$ is a constant. Noting that $\left(g_{0}^{2}\right)^{\prime}=2 g_{0} g_{0}^{\prime}$ also vanishes at the left boundary, it follows that $C_{2}=0$ and

$$
g_{0}^{\prime}+\alpha g_{0}=\sqrt{\alpha} .
$$


Imposing the left side boundary condition that $g_{0}(0)=0$, the solution of this first-order ODE is given by

$$
g_{0}\left(\xi_{0}\right)=\frac{1}{\sqrt{\alpha}}\left(1-e^{-\alpha \xi_{0}}\right) .
$$

2.3. Composite solution. We now generate the composite solution by matching the outer limit of the inner expansion with the inner limit of the outer expansion [6]. The superscript $o l$ on $g_{0}$ denotes the outer limit of the zeroth-order inner expansion. Similarly, the superscript $i l$ on $f_{0}$ stands for the inner limit of the zeroth-order outer expansion. For the outer expansion,

$$
f_{0}^{i l}\left(\xi_{0}\right)=\lim _{\substack{t \rightarrow \infty \\ \xi_{0} \text { fixed }}} f_{0}\left(\eta_{\ell 0}+t^{-\frac{1}{2}} \xi_{0}\right) .
$$

With $\eta>\eta_{\ell 0}$, the outer limit of the inner expansion is

$$
g_{0}^{o l}(\eta)=\lim _{\substack{t \rightarrow \infty \\ \eta \text { fixed }}} g_{0}\left(\left(\eta-\eta_{\ell 0}\right) t^{\frac{1}{2}}\right) .
$$

The expansion is consistent since

$$
f_{0}^{i l}\left(\xi_{0}\right)=g_{0}^{o l}(\eta)=\frac{1}{\sqrt{\alpha}} .
$$

Hence we can construct the leading-order composite solution as

$$
u_{0}(x, t)=\frac{1}{\sqrt{t}}\left(f_{0}(\eta)+g_{0}\left(\xi_{0}\right)-\frac{1}{\sqrt{\alpha}}\right) .
$$

Reexpressed in terms of the original variables, we have

$$
u_{0}(x, t)=-\frac{x}{2 \alpha t}-\frac{1}{\sqrt{\alpha t}} e^{-\alpha(x+2 \sqrt{\alpha t})},
$$

which is valid only for the range of $x$ values for which $u_{0}(x, t) \geq 0$. As an addendum, we note that

$$
u_{0}(-2 \sqrt{\alpha t}, t)=0
$$

and

$$
u_{0}(0, t)=-\frac{1}{\sqrt{\alpha t}} e^{-2 \alpha \sqrt{\alpha t}} .
$$

For the leading-order $x_{\ell}$ and $x_{r}$, the left boundary condition is satisfied exactly, and the right boundary condition is satisfied to exponentially small terms. But the solution itself is valid only to $\mathcal{O}\left(\frac{1}{\sqrt{t}}\right)$. The left and the right boundaries in $x$ are correct to $\mathcal{O}(\sqrt{t})$. Thus, the support of the solution is consistent with the leading-order left and right boundaries.

3. Higher-order solution. As an extension of the above results, we wish to construct high-order approximations of the late-time outer and inner solutions. These are respectively

and

$$
u^{\mathbf{o}}(x, t)=\frac{1}{\sqrt{t}} f(\eta), \quad \eta=\frac{x}{\sqrt{t}}
$$

$$
u^{\mathbf{i}}(x, t)=\frac{1}{\sqrt{t}} g(\xi), \quad \xi=\left(\eta-\eta_{\ell}(t)\right) \sqrt{t} .
$$


Here $f$ and $g$ are two bounded $\mathcal{O}(1)$ functions and $\eta_{\ell}$ denotes the left boundary. Similarly $\eta_{r}$ is the position of the right boundary.

We now expand the solution and its two moving boundaries in terms of gauge functions $\nu_{n}(t)$ as

$$
\begin{aligned}
f(\eta) & =f_{0}(\eta)+\nu_{1}(t) f_{1}(\eta)+\nu_{2}(t) f_{2}(\eta)+\cdots, \\
g(\xi) & =g_{0}(\xi)+\nu_{1}(t) g_{1}(\xi)+\nu_{2}(t) g_{2}(\xi)+\cdots, \\
\eta_{\ell}(t) & =\eta_{\ell 0}+\nu_{1}(t) \eta_{\ell 1}+\nu_{2}(t) \eta_{\ell 2}+\cdots \\
\eta_{r}(t) & =\eta_{r_{0}}+\nu_{1}(t) \eta_{r 1}+\nu_{2}(t) \eta_{r_{2}}+\cdots,
\end{aligned}
$$

where $\nu_{1}(t) \rightarrow 0$ and $\nu_{2}(t) / \nu_{1}(t) \rightarrow 0$ as $t \rightarrow \infty$. The leading-order terms of these expansions have been presented in the previous section. We are now interested in deriving the next-order corrections of these expansions. In a subtle way, we regard $g$ to be dependent on $\xi$, defined in terms of $\eta_{\ell}$ as opposed to $\eta_{\ell 0}$. We know that this is true if the expansion is truncated to the first term. For the order of asymptotics of interest, denoted by $N$, it is implied that $\xi$ will be regarded as $\xi_{0 N}$, where

$$
\xi_{0 N}=\left(\eta-\eta_{\ell 0}-\sum_{1}^{N} \eta_{\ell_{i}} \nu_{i}(t)\right) \sqrt{t} .
$$

3.1. Higher-order outer solution. Substituting (3.1) and (3.3) into the original PDE and keeping all the known terms up to $\mathcal{O}\left(\frac{1}{t^{2}}\right)$, we obtain

$$
\frac{\nu_{1}}{2 t} \eta f_{1}^{\prime}+\left(\nu_{1}^{\prime}+\frac{\nu_{1}}{2 t}\right) f_{1}=\frac{1}{4 \alpha^{2} t^{3 / 2}} .
$$

For a distinguished limit, since $f_{1}=\mathcal{O}(1)$, we impose

$$
\nu_{1}^{\prime}+\frac{\nu_{1}}{2 t}=\frac{1}{4 \alpha^{2} t^{3 / 2}} .
$$

The solution of this first-order ODE is given by

$$
\nu_{1}(t)=\frac{\ln t}{4 \alpha^{2} \sqrt{t}}+\frac{C_{3}}{\sqrt{t}},
$$

where $C_{3}$ is an arbitrary constant.

This expression suggests that the next-order gauge function consists of two parts, namely

and

$$
\nu_{1,1}(t)=\frac{\ln t}{\sqrt{t}}
$$

$$
\nu_{1,2}(t)=\frac{1}{\sqrt{t}} .
$$

Note that, alternatively, if we were to balance the right-hand side of (3.8) with the first term on the left-hand side, we would get $\nu_{1}(t)=\frac{1}{2 \alpha^{2} \sqrt{t}}$, a term that is already captured by $\nu_{1,2}(t)$. This observation confirms that the appropriate distinguished limit is indeed given by (3.9).

As per (3.11) and (3.12), we now rewrite (3.3) as

$$
f(\eta)=f_{0}(\eta)+\frac{\ln t}{\sqrt{t}} f_{1,1}(\eta)+\frac{1}{\sqrt{t}} f_{1,2}(\eta)+\cdots,
$$


where $f_{1,1}$ and $f_{1,2}$ are two $\mathcal{O}(1)$ functions to be determined. Substituting (3.13) into the original PDE, while matching the $\mathcal{O}\left(\frac{\ln t}{t^{2}}\right)$ terms only, we arrive at the first-order ODE

$$
2 \alpha\left(f_{0} f_{1,1}\right)^{\prime}+\frac{\eta}{2} f_{1,1}^{\prime}+f_{1,1}=0 .
$$

Substituting $-\eta /(2 \alpha)$ for $f_{0}$, we get

$$
\frac{\eta}{2} f_{1,1}^{\prime}=0
$$

which amounts to

$$
f_{1,1}(\eta)=C_{4}
$$

where $C_{4}$ is a constant. The ODE for $f_{1,2}$ is obtained by balancing terms up to $\mathcal{O}\left(\frac{1}{t^{2}}\right)$. This gives

$$
2 \alpha\left(f_{0} f_{1,2}\right)^{\prime}+\frac{\eta}{2} f_{1,2}^{\prime}+f_{1,2}-f_{1,1}+\frac{1}{2}\left(f_{0}^{2}\right)^{\prime \prime}=0 .
$$

Substituting the expressions for $f_{0}$ and $f_{1,1}$ into the above equation, the latter simplifies to

$$
\frac{\eta}{2} f_{1,2}^{\prime}=\frac{1}{4 \alpha^{2}}-C_{4}
$$

so that

$$
f_{1,2}(\eta)=2\left(\frac{1}{4 \alpha^{2}}-C_{4}\right) \ln \eta+C_{5},
$$

where $C_{5}$ is a constant. Since $\eta=0$ is within the domain of the solution,

$$
C_{4}=\frac{1}{4 \alpha^{2}} \text {. }
$$

It follows that

$$
f_{1,2}(\eta)=C_{5}
$$

Hence we have shown that

$$
f(\eta)=-\frac{\eta}{2 \alpha}+\frac{\ln t}{4 \alpha^{2} \sqrt{t}}+\frac{C_{5}}{\sqrt{t}}+\cdots,
$$

and solving for the $\eta$ value where the above expression vanishes, we obtain

$$
\eta_{r}(t)=\frac{\ln t}{2 \alpha \sqrt{t}}+\frac{2 \alpha C_{5}}{\sqrt{t}}+\cdots .
$$

In terms of the original variables, we have

$$
u^{\mathbf{o}}(x, t)=-\frac{x}{2 \alpha t}+\frac{\ln t}{4 \alpha^{2} t}+\frac{C_{5}}{t}+\cdots
$$

and

$$
x_{r}(t)=\frac{\ln t}{2 \alpha}+2 \alpha C_{5}+\cdots .
$$


3.2. Exact solution. It is worth noting that the truncated outer solution of (3.24) is in fact an exact solution to the original PDE. A simple way to realize this is to seek a solution of the form

$$
u(x, t)=-\frac{x}{2 \alpha t}+w(t),
$$

where $w$ is unknown. Substitution of this expression into (1.1) leads to

$$
w^{\prime}+\frac{w}{t}=\frac{1}{4 \alpha^{2} t^{2}} .
$$

This ODE can be solved explicitly, yielding

$$
w(t)=\frac{\ln t}{4 \alpha^{2} t}+\frac{C_{6}}{t},
$$

where $C_{6}$ is a constant. Combining (3.26) and (3.28), we get the same expression as (3.24) for the outer solution with $C_{5}$ replacing $C_{6}$.

3.3. Inner solution. We now consider the higher-order expansion of the inner solution. From the results of the higher-order gauge function in (3.11) and (3.12), we construct

$$
g(\xi)=g_{0}(\xi)+\frac{\ln t}{\sqrt{t}} g_{1,1}(\xi)+\frac{1}{\sqrt{t}} g_{1,2}(\xi)+\cdots
$$

where $\xi=\left(\eta-\eta_{\ell}(t)\right) \sqrt{t}$

$$
\eta_{\ell}(t)=-2 \sqrt{\alpha}+\frac{\ln t}{\sqrt{t}} \eta_{\ell 1,1}+\frac{1}{\sqrt{t}} \eta_{\ell 1,2}+\cdots,
$$

and

$$
g_{1,1}(0)=0, \text { and } g_{1,2}(0)=0 .
$$

Using (3.29) in the PDE of (1.1) and retaining only the $\mathcal{O}\left(t^{-1}\right)$ terms, we obtain the solution

$$
g_{0}(\xi)=\frac{1}{\sqrt{\alpha}}\left(1-e^{-\alpha \xi}\right),
$$

where the leading-order solution is represented in terms of $\xi$ rather than $\xi_{0}$, an important distinction. We recall that $\xi_{0 N}$ is used in lieu of $\xi$ as given in (3.7).

The next order is obtained by matching terms $\mathcal{O}\left(t^{-3 / 2} \ln t\right)$ to get the differential equation

$$
\sqrt{\alpha} g_{1,1}^{\prime}=2 \alpha\left(g_{0} g_{1,1}\right)^{\prime}+\left(g_{0} g_{1,1}\right)^{\prime \prime}
$$

This ODE can be rearranged as

$$
g_{0} g_{1,1}^{\prime \prime}+\left(2 \alpha g_{0}+2 g_{0}^{\prime}-\sqrt{\alpha}\right) g_{1,1}^{\prime}+\left(g_{0}^{\prime \prime}+2 \alpha g_{0}^{\prime}\right) g_{1,1}=0 .
$$

Utilizing the exact form of $g_{0}$ given by (3.32), and recognizing that $g_{0}^{\prime}+\alpha g_{0}=\sqrt{\alpha}$, the above equation simplifies to

$$
\left(1-e^{-\alpha \xi}\right) g_{1,1}^{\prime \prime}+\alpha g_{1,1}^{\prime}+\alpha^{2} e^{-\alpha \xi} g_{1,1}=0 .
$$

This second-order linear ODE can be solved explicitly, by first observing that $\xi \mapsto e^{-\alpha \xi}$ is a solution and then, by applying the method of variation of parameters to find a second independent solution. It follows that

$$
g_{1,1}(\xi)=C_{7} e^{-\alpha \xi}+C_{8}\left(1+e^{-\alpha \xi} \ln \left(e^{\alpha \xi}-1\right)\right),
$$


where $C_{7}$ and $C_{8}$ are arbitrary constants. Since the second part of the solution diverges at $\xi=0, C_{8}=0$. The boundary condition $g_{1,1}(0)=0$ implies that $C_{7}=0$. Thus,

$$
g_{1,1}(\xi)=0 .
$$

Before proceeding further for evaluating $g_{1,2}$, we need to determine $\eta_{\ell 1,1}$ and other constraints if any, by imposing an intermediate matching condition between the outer and inner solutions that have been obtained so far. We define

$$
f^{i}(\xi)=f\left(\eta_{\ell}(t)+\xi / \sqrt{t}\right) .
$$

Using (3.22) and (3.30), we obtain

$$
f^{i}(\xi)=\frac{1}{\sqrt{\alpha}}+\left(1-2 \alpha \eta_{\ell 1,1}\right) \frac{\ln t}{4 \alpha^{2} \sqrt{t}}+\mathcal{O}\left(t^{-1 / 2}\right) .
$$

Similarly, we define

$$
g^{o}(\eta)=g\left(\left(\eta-\eta_{\ell}(t)\right) \sqrt{t}\right)
$$

and get

$$
g^{o}(\eta)=\frac{1}{\sqrt{\alpha}}\left(1-e^{-\alpha\left(\eta-\eta_{\ell}(t)\right) \sqrt{t}}\right)+\mathcal{O}\left(t^{-1 / 2}\right) .
$$

For matching as $t \rightarrow \infty$, since $\eta>\eta_{\ell}(t)$, we have

$$
g^{o}(\eta)=\frac{1}{\sqrt{\alpha}}+\mathcal{O}\left(t^{-1 / 2}\right) .
$$

Therefore, intermediate matching between inner and outer solutions requires that

$$
1-2 \alpha \eta_{\ell 1,1}=0 \Rightarrow \eta_{\ell 1,1}=\frac{1}{2 \alpha} .
$$

A composite solution may also be constructed at this order, as given by

$$
u_{01,1}(x, t)=-\frac{x}{2 \alpha t}+\frac{\ln t}{4 \alpha^{2} t}-\frac{1}{\sqrt{\alpha t}} e^{-\alpha\left(x+2 \sqrt{\alpha t}-\frac{1}{2 \alpha} \ln t\right)},
$$

where the subscript 01,1 implies inclusion from zeroth-order up to $\nu_{1,1}$ terms. As in the leading-order solution, this expression is exactly zero at $x=-2 \sqrt{\alpha t}+\frac{\ln t}{2 \alpha}$, while it yields an exponentially decaying term at $x=\frac{\ln t}{2 \alpha}$. Essentially, this first-order correction is a shift applied to the zeroth-order solution. In particular, the evaluation of the mass balance

leads to

$$
I_{01,1}=\int_{-2 \sqrt{\alpha t}+\frac{\ln t}{2 \alpha}}^{\frac{\ln t}{2 \alpha}} u_{01,1}(x, t) d x
$$

$$
I_{01,1}=1+\frac{1}{\alpha^{3 / 2} \sqrt{t}}\left(e^{-2 \alpha \sqrt{\alpha t}}-1\right)=1+\mathcal{O}\left(t^{-1 / 2}\right),
$$

which shows that mass conservation is verified consistently with the present order of approximation.

Next, matching the $\mathcal{O}\left(t^{-3 / 2}\right)$ terms of the expansion, we arrive at

$$
-\frac{1}{2} g_{0}-\eta_{\ell 1,1} g_{0}^{\prime}+\sqrt{\alpha} g_{1,2}^{\prime}=2 \alpha\left(g_{0} g_{1,2}\right)^{\prime}+\left(g_{0} g_{1,2}\right)^{\prime \prime} .
$$

We can now substitute the value of $\eta_{\ell 1,1}$ into (3.47). This reduces the equation to

$$
\left(1-e^{-\alpha \xi}\right) g_{1,2}^{\prime \prime}+\alpha g_{1,2}^{\prime}+\alpha^{2} e^{-\alpha \xi} g_{1,2}=-\frac{1}{2} .
$$


This differential equation is identical to that of $g_{1,1}$ except for the nonhomogeneous part. Therefore the homogeneous solution of this ODE is given by (3.36) and, applying the method of variation of parameters, we obtain a particular solution as

$$
g_{1,2}^{*}(\xi)=\frac{1}{2 \alpha^{2}}\left\{1-\alpha \xi+e^{-\alpha \xi} \operatorname{Li}_{2}\left(1-e^{\alpha \xi}\right)\right\}
$$

where $\mathrm{Li}_{2}$ is the dilogarithm function defined by

$$
\operatorname{Li}_{2}(z)=\int_{z}^{0} \frac{\ln (1-y)}{y} d y .
$$

The next-order correction to the inner solution may be expressed as

$$
g_{1,2}(\xi)=C_{9} e^{-\alpha \xi}+C_{10}\left(1+e^{-\alpha \xi} \ln \left(e^{\alpha \xi}-1\right)\right)+\frac{1}{2 \alpha^{2}}\left\{1-\alpha \xi+e^{-\alpha \xi} \operatorname{Li}_{2}\left(1-e^{\alpha \xi}\right)\right\},
$$

where $C_{9}$ and $C_{10}$ are constants. Recalling that the second term is incompatible with the left boundary condition and must therefore be zero, and using the condition that $g_{1,2}=0$ at $\xi=0$, we obtain that $C_{10}=0$ and $C_{9}=-\frac{1}{2 \alpha^{2}}$. Therefore

$$
g_{1,2}(\xi)=\frac{1}{2 \alpha^{2}}\left\{1-e^{-\alpha \xi}-\alpha \xi+e^{-\alpha \xi} \operatorname{Li}_{2}\left(1-e^{\alpha \xi}\right)\right\} .
$$

We now apply the higher-order matching condition between the inner and outer expansions. Using the definitions (3.38) and (3.40) with $\eta_{\ell}(t)$ given by (3.30), we write

$$
f^{i}(\xi)=\frac{1}{\sqrt{\alpha}}-\frac{\xi}{2 \alpha \sqrt{t}}+\left(C_{5}-\frac{\eta_{\ell 1,2}}{2 \alpha}\right) \frac{1}{\sqrt{t}}+o\left(t^{-1 / 2}\right)
$$

and

$$
g^{o}(\eta)=\frac{1}{\sqrt{\alpha}}\left(1-e^{-\alpha\left(\eta-\eta_{\ell}(t)\right) \sqrt{t}}\right)+\frac{1}{\sqrt{t}} g_{1,2}\left(\left(\eta-\eta_{\ell}(t)\right) \sqrt{t}\right)+\cdots
$$

We consider as before the limiting expressions for $t \rightarrow \infty, \xi$ fixed for $f^{i}(\xi)$ and $\eta$ fixed with $\eta>\eta_{\ell}(t)$ for $g^{o}(\eta)$. The limit up to $\mathcal{O}(1 / \sqrt{t})$ is considered. These limits are denoted as $f^{i l}$ for $f^{i}$ and $g^{o l}$ for $g^{o}$. It is easy to work out that

$$
f^{i l}(\xi)=\frac{1}{\sqrt{\alpha}}-\frac{\xi}{2 \alpha \sqrt{t}}+\left(C_{5}-\frac{\eta_{\ell 1,2}}{2 \alpha}\right) \frac{1}{\sqrt{t}}
$$

and

$$
g^{o l}(\eta)=\frac{1}{\sqrt{\alpha}}-\frac{\eta-\eta_{\ell}(t)}{2 \alpha}+\frac{1}{2 \alpha^{2} \sqrt{t}} .
$$

The first two terms match automatically. To match the last term we require that

$$
C_{5}-\frac{\eta_{\ell 1,2}}{2 \alpha}=\frac{1}{2 \alpha^{2}} \Rightarrow \eta_{\ell 1,2}=2 \alpha C_{5}-\frac{1}{\alpha} .
$$

Finally, we construct the new composite solution by adding $f(\eta)$ and $g(\xi)$, and then subtracting $f^{i l}$ or $g^{o l}$. In a compact form, we obtain

$$
u(x, t)=\frac{x_{r}(t)-x}{2 \alpha t}-\left[\frac{1}{\sqrt{\alpha t}}+\frac{1}{2 \alpha^{2} t}\left(1-\operatorname{Li}_{2}\left(1-e^{\alpha\left(x-x_{\ell}(t)\right)}\right)\right)\right] e^{\alpha\left(x_{\ell}(t)-x\right)}+\cdots,
$$

where

$$
x_{\ell}(t)=-2 \sqrt{\alpha t}+\frac{1}{2 \alpha} \ln t+2 \alpha C_{5}-\frac{1}{\alpha}+\cdots
$$


and

$$
x_{r}(t)=\frac{1}{2 \alpha} \ln t+2 \alpha C_{5}+\cdots .
$$

The concise representation of the solution in terms of the two boundaries illustrates that $u\left(x_{\ell}(t), t\right)=0$ and $u\left(x_{r}(t), t\right)$ is exponentially small for $t \rightarrow \infty$. We point out that the constant $C_{5}$ cannot be determined in the general case. However, the value of this constant may be obtained numerically for a particular choice of initial data. With an impulse-like initial data our numerical estimates shown below indicate a value close to unity for $2 \alpha C_{5}$. Our conjecture is that the above solution converges to the true solution as $t \rightarrow \infty$ for any $L^{p}$-norm.

4. Numerical experiments. To verify the validity of our results, we solve (1.1) numerically for the following initial condition:

$$
u_{I}(x)= \begin{cases}\frac{1}{2 \epsilon}, & |x+\epsilon| \leq \epsilon, \\ 0, & |x+\epsilon|>\epsilon,\end{cases}
$$

where $\epsilon>0$ is a constant. For good stability properties we apply a fully implicit scheme with first-order upwind discretization for the convective term and central discretization for the diffusive term. If a uniform spatial step size and adaptive time stepping are employed, the discrete form of (1.1) reads

$$
u_{j}^{n+1}=u_{j}^{n}+\alpha \frac{\Delta t_{n}}{\Delta x}\left[\left(u_{j+1}^{n+1}\right)^{2}-\left(u_{j}^{n+1}\right)^{2}\right]+\frac{\Delta t_{n}}{2 \Delta x^{2}}\left[\left(u_{j+1}^{n+1}\right)^{2}-2\left(u_{j}^{n+1}\right)^{2}+\left(u_{j-1}^{n+1}\right)^{2}\right] .
$$

A comparison between the numerical and analytical results is shown in Fig. 1 for $\alpha=2$, and $\epsilon=0.01$. In the simulation, $\Delta x=0.005$ and $\max \left(\Delta t_{n}\right)=0.01$. It is remarkable that with a pulse initial data, the analytical results are able to generate the complete solution with a high level of accuracy. In Fig. 2 the inner, outer and composite solutions are shown for $t=10$ and $\alpha=2$. The subscript 01,2 implies inclusion up to $\nu_{1,2}$ terms. We point out that $u_{01,2}$ is the same as $g_{01,2}$.

5. Conclusion. We have derived late-time asymptotic solutions to a nonlinear advective-diffusion equation that has applications in porous media flow. In our particular case, it describes the thickness of a buoyancy driven plume in an inclined bed. With a compactly supported initial condition, the leading-order outer solution also satisfies the hyperbolic problem with a pulse initial condition. The next-order correction for the outer solution is purely time dependent, and at this order the partial differential equation is satisfied exactly. Only a moving left boundary layer is required to generate the composite solution. We have obtained the composite solution to orders $1 / \sqrt{t},(1 / t) \ln t$, and $1 / t$. It is shown that an unknown constant appears in the solution at $\mathcal{O}(1 / t)$. 


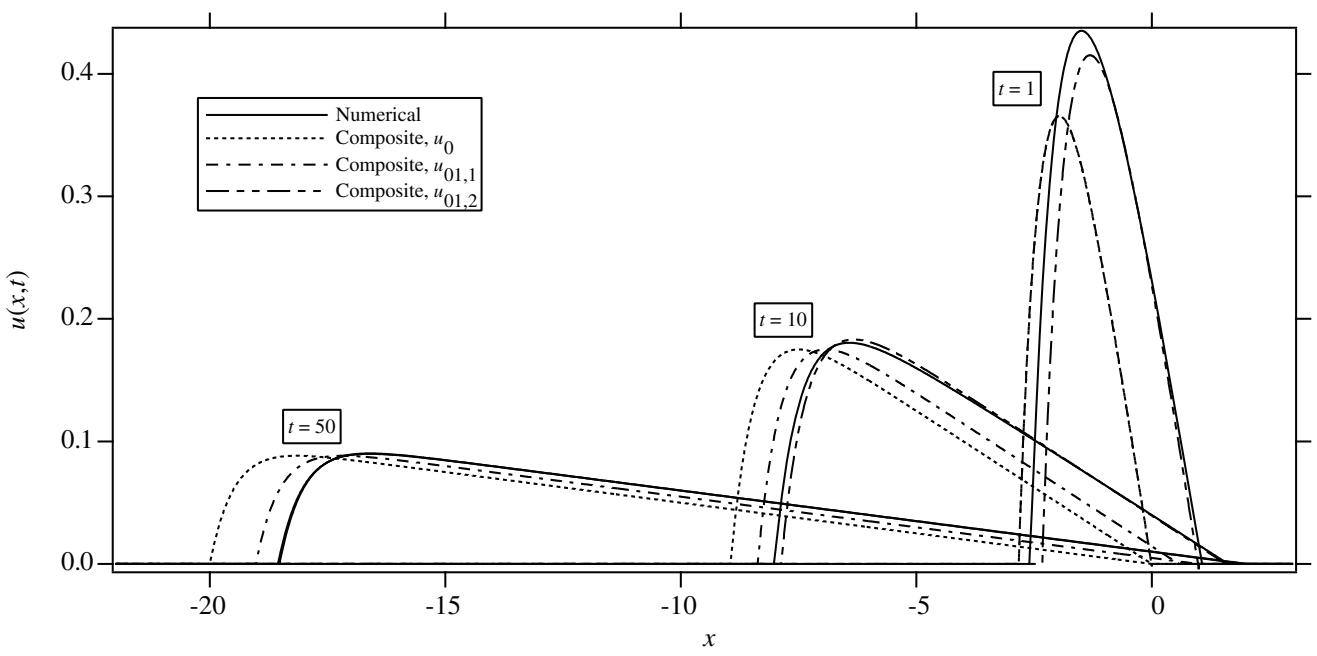

FIG. 1. Asymptotic and analytical solutions for $t=1,10$ and 50, with $\alpha=2$. Note that for $t=1$, the $\nu_{1,1}$ term is zero and $u_{01,1}(x, 1)=u_{0}(x, 1)$. For $t=50$, the numerical and the higherorder analytical results with $2 \alpha C_{5}=1$ are almost the same.

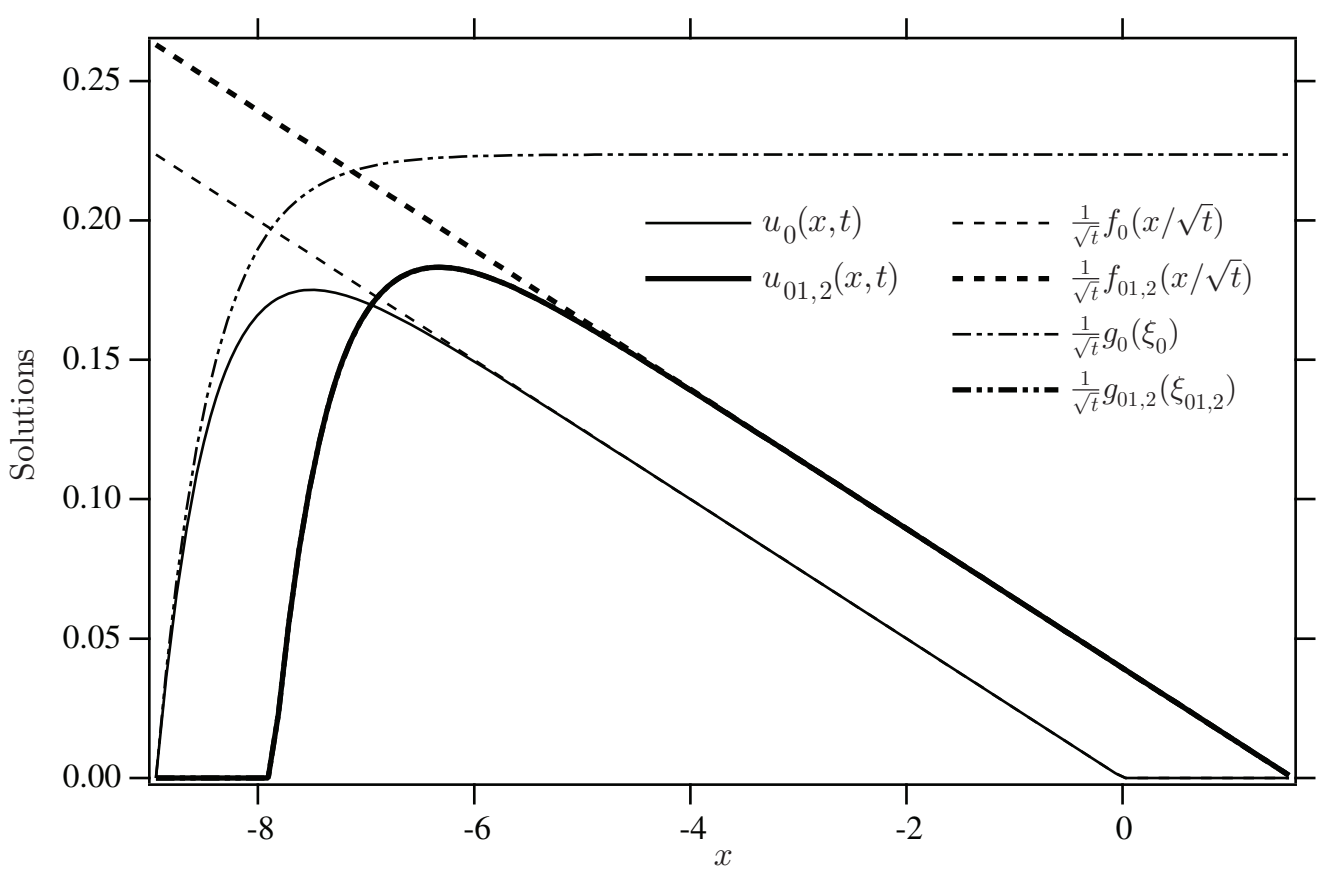

FIG. 2. Asymptotic inner, outer, and composite solutions $(\alpha=2, t=10)$. 


\section{REFERENCES}

[1] G. I. Barenblatt, Scaling, Self-Similarity, and Intermediate Asymptotics, Cambridge University Press (1996). MR1426127 (98a:00005)

[2] B. H. Gilding and L. A. Peletier, The Cauchy problem for an equation in the theory of infiltration, Arch. Rational Mech. Anal., 61 (1976), 127-140. MR0408428 (53:12192)

[3] B. H. Gilding, Properties of Solutions of an Equation in the Theory of Infiltration, Arch. Rational Mech. Anal., 65 (1977), 203-225. MR0447847 (56:6157)

[4] B.H. Gilding, The occurrence of interfaces in nonlinear diffusion-advection processes, Arch. Rational Mech. Anal. 100 (1988), 243-263. MR.918796 (89f:35104)

[5] R. E. Grundy, Asymptotic Solution of a Model Nonlinear Convective Diffusion Equation, IMA J. Appl. Math., 31 (1983), 121-137. MR.728117 (85g:76024)

[6] J. Kevorkian and J. D. Cole, Perturbation Methods in Applied Mathematics, Springer-Verlag (1981). MR608029 (82g:34082)

[7] Ph. Laurençot and F. Simondon, Long-time behaviour for porous medium equations with convection, Proc. Roy. Soc. Edinburgh Sect. A, 128 (1998), 315-336. MR1621331 (99f:35108)

[8] Ph. Laurençot, Long-Time Behaviour for Diffusion Equations with Fast Convection, Annali di Matemetica pura ed applicata, 175 (1998), 233-251. MR1748225 (2001b:35173)

[9] R. de Loubens and T. S. Ramakrishnan, Analysis and Computation of Gravity Induced Migration in Porous Media, accepted for publication (2010).

[10] D. A. Sapronov and A. E. Shishkov, Asymptotic behaviour of supports of solutions of quasilinear multidimensional parabolic equations of nonstationary diffusion-convection type, Sb. Math. 197 (2006), 753-790. MR2264331 (2007f:35168)

[11] V. Vanaja and P. L. Sachdev, Asymptotic Solutions of a Generalized Burgers Equation, Quarterly of Appl. Math., 50 (1992), 627-640. MR.1193660 (93h:35184) 